\title{
The impact of thyroid autoantibodies on the cycle outcome and embryo quality in women undergoing intracytoplasmic sperm injection
}

\author{
Vehbi Yavuz Tokgoz (10, Berker Isim and Ahmet Basar Tekin
}

\begin{abstract}
Background: The influence of thyroid autoimmunity in in-vitro fertilization/intracytoplasmic sperm injection (ICSI) cycles is still a controversial issue. Although some women are euthyroid, some studies found thyroid dysfunction and thyroid autoimmunity increased the incidence of infertility. We aimed to evaluate the impact of anti-thyroid antibodies on the cycle parameters, embryo quality, and pregnancy outcomes in women who underwent ICSI cycles.

Results: Fertilization rate was significantly higher in anti-thyroid antibody-negative patients than in anti-thyroid antibody-positive patients (97.1 \pm 10.5 vs. $91.5 \pm 19.8$, respectively, $p=0.003$ ). Clinical pregnancy, miscarriage, and ongoing pregnancy rates were not different among study groups. The presence of top-quality embryo was lower in anti-thyroid antibody-positive patients but it did not reach any statistical significance (56.5\% vs. $67.8 \%, p=0.09)$. In the subgroup analysis, there were no significant differences except for the miscarriage rate according to the TSH threshold.

Conclusion: The clinical pregnancy and ongoing pregnancy were not affected by the positivity of anti-thyroid antibodies. We showed that thyroid autoimmunity may have an impact on fertilization rate and embryo quality in patients undergoing ICSI cycles. Miscarriage rate also increases with thyroid autoimmunity and TSH levels above $2.5 \mu \mathrm{IU} / \mathrm{L}$.
\end{abstract}

Keywords: Thyroid, Autoantibody, Autoimmunity, Intracytoplasmic sperm injection, Embryo quality

\section{Background}

Thyroid dysfunction may have an essential impact on reproductive outcomes. In particular, subclinical hypothyroidism is important in association with infertility and pregnancy outcome after assisted reproduction technologies (ART) [1]. Thyroid autoimmunity is the most common cause of subclinical hypothyroidism, and it can make an effect on fertility potential changing peripheral estrogen metabolism [2]. The prevalence of subclinical hypothyroidism is approximately 2 to $4 \%$ [3] and that of thyroid autoimmunity ranges from 5 to $20 \%$ [4], but it may reach up to $25 \%$ in infertile women [5]. Thyroid dysfunction is more frequent in women

\footnotetext{
* Correspondence: mdtokgoz@hotmail.com

Department of Obstetrics and Gynecology, Eskisehir Osmangazi University School of Medicine, 26100 Eskisehir, Turkey
}

who have thyroid autoimmunity [6]. Association of improper functioning of thyroid gland and in vitro fertilization (IVF) outcome is an important issue because supraphysiological estradiol levels affect thyroid-binding globulin and also TSH (thyroid-stimulating hormone) levels [1]. This effect may have some negative impact on pregnancy outcome by triggering subclinical hypothyroidism, especially in IVF cycles. Thyroid function evaluation of infertile women might provide some helpful information, and if necessary, treatment may improve pregnancy outcome of ART. So, routine thyroid evaluation including anti-thyroid autoantibodies (ATA) such as anti-thyroglobulin antibody (Anti-TG) and anti-thyroid peroxidase antibody (Anti-TPO) was suggested in infertile women [7]. Although some women are euthyroid, some studies showed that ATA increased the incidence 
of infertility [6]. The presence of ATA was also associated with an increased miscarriage rate $[8,9]$ However, there are some controversial results regarding the effect of thyroid autoimmunity on IVF cycle outcome even in euthyroid women. A previous study demonstrated that the presence of ATA did not affect the miscarriage rate among ART patients [10]. On the contrary, Toulis et al. showed that the risk of spontaneous miscarriage rate increased with ATA positivity in women who conceive through IVF [9]. Some studies that have investigated the effect of ATA on the clinical pregnancy rate did not establish any significant association between the ATA positivity and clinical pregnancy outcome in euthyroid women undergoing ART $[10,11]$, but others observed a significant reduction in pregnancy rates with thyroid autoimmunity [12]. Embryo quality is an important factor in IVF success, and the relationship of embryo quality with thyroid autoimmunity has not been identified yet. Recently, two studies have suggested the negative impact of ATA on embryo quality in euthyroid patients undergoing IVF/ICSI (intracytoplasmic sperm injection) cycles $[13,14]$.

Thus, the present study aimed to evaluate the effect of ATA on the cycle outcome, embryo quality, and pregnancy outcome of ICSI cycles in women with normal thyroid function.

\section{Methods}

We conducted a retrospective cohort study investigating 474 patients who underwent assisted reproduction cycles between January 2018 and May 2019 at an infertility unit of university-based hospital. The study was approved by the Instutional Review Board, and the principles of Helsinki Declaration were followed. IVF/ICSI cycles were performed for couples who had unexplained infertility, tubal factor infertility, polycystic ovarian syndrome, and male factor infertility. Autoimmune diseases, lupus anticoagulant, hyperprolactinemia, severe liver, or renal dysfunction were excluded. Women who had TSH levels $>4.0 \mathrm{IU} / \mathrm{mL}$ were also excluded.

Demographic characteristics and basal parameters were determined. Basal hormone levels such as FSH (follicle-stimulating hormone), LH (luteinizing hormone), estradiol, AMH (anti-mullerian hormone), TSH, free $\mathrm{T}_{3}$, free $\mathrm{T}_{4}$, and anti-thyroid autoantibodies (ATA) (anti-TG and anti-TPO) were obtained at the day 2 or 3 of the menstrual cycle before starting the ovarian stimulation. Patient's serum samples were measured for FSH, LH, and TSH by electrochemiluminescent immunometric assay using commercial kits (Cobas 6000, e601, Roche Diagnostics Poland). Serum AMH concentrations were analyzed by enzyme-linked immunosorbent assay ELISA (Diagnostic Systems Laboratories, Webster, TX). Electrochemiluminescence immunoassay (ECLIA) method was used for thyroid antibodies, and normal values were accepted as $<35 \mathrm{IU} / \mathrm{mL}$ and $<115 \mathrm{IU} / \mathrm{mL}$ for anti-TPO and anti-TG antibodies, respectively.

$\mathrm{GnRH}$ antagonist protocol was performed for all patients. Ovarian stimulation was initiated with recombinant FSH (Gonal-f, Merck, Germany) starting on the cycle day 2 or 3 . The doses of gonadotropins were adjusted according to the patient's age, BMI, and basal antral follicle count. GnRH antagonist (Cetrorelix, Merck, Germany) was administered if leading follicle reached $14 \mathrm{~mm}$ or serum estradiol level exceeded the $300 \mathrm{pg} / \mathrm{mL}$ and continued until the hCG administration. When the dominant follicle reached $18 \mathrm{~mm}$ in diameter, ovulation trigger was achieved with the administration of recombinant hCG (Ovitrelle $250 \mu$ g; Merck, Germany). Oocyte retrieval was performed by transvaginal ultrasonography $36 \mathrm{~h}$ after triggering. ICSI was performed in all cases as a standard procedure. Fertilization was evaluated $16-18 \mathrm{~h}$ after ICSI procedure and cleavage observation was checked $48-72 \mathrm{~h}$ after oocyte pick-up. Embryo development was observed, and embryo quality was determined according to the guidelines $[15,16]$. According to the embryonic development, day 3 (cleavage stage) or day 5 (blastocyst stage) fresh embryo transfer was performed under the ultrasound guidance. Luteal phase was supported by the administration of vaginal progesterone gel (Crinone $8 \%$ gel, Merck, Germany) until the ninth gestational week.

Implantation was defined by assessing the positive test result for serum beta-hCG levels 12 days after embryo transfer. The clinical pregnancy was determined as the presence of a fetal heartbeat. Miscarriage was defined as a loss of pregnancy before 25 weeks of gestation. Ongoing pregnancy was defined as pregnancies that reached 25week gestation or more.

Statistical evaluation was performed by the Statistical Package for the Social Sciences (SPSS) Version 20.0 (SPSS Inc., Chicago, IL). Demographic parameters and cycle outcomes were analyzed with mean \pm SD (standard deviation) and median values. Kolmogorov-Smirnov test was used to evaluate the distribution of the parameters. Normally distributed data were analyzed by using Student's $t$ test. Mann-Whitney $U$ and Kruskal-Wallis test were performed in case of non-parametric continuous and categorical data. The frequency and percentages values were expressed for categorical data, and Pearson chi-square test or Fisher Exact test was used to compare the variables. We also used univariate and multivariate logistic regression analysis to determine the effect of potential confounders on cycle outcomes. Odds ratio (OR) with $95 \%$ confidence interval (CI) expressed the effects of covariates. A $p$ value of $<0.05$ was considered statistically significant.

\section{Results}

A total of 474 patients were evaluated initially. Of these, 315 patients who have met the inclusion criteria were 
analyzed. Patients were divided into two groups according to the presence of at least one anti-thyroid autoantibody. Two hundred and fifty patients (79.3\%) had no ATA, and 65 patients $(20.7 \%)$ had at least one ATA positivity. There were no significant differences for basal and demographic parameters between the main groups (Table 1). Only free $\mathrm{T}_{4}$ levels were significantly higher in the ATA-positive cases. Cycle outcomes except fertilization rate were also similar between ATA-negative and ATA-positive patients (Table 2). Overall implantation rate (IR), clinical pregnancy rate (CPR), miscarriage rate (MR), and ongoing pregnancy rate were $50.5 \%, 43.5 \%, 10.1 \%$, and $30.7 \%$, respectively. $\mathrm{CPR}$ and ongoing pregnancy rate were also comparable between study groups (Fig. 1). Among ATA-positive women, $19(29.3 \%)$ had only anti-TG positivity, $22(33.8 \%)$ had only anti-TPO positivity, and 24(36.9\%) women had both antibodies positivity. We also evaluated the impact of ATA positivity on pregnancy outcomes with subgroup analysis for TSH level as below and above $2.5 \mathrm{IU} / \mathrm{L}$ (Table 3). There was no significant difference regarding the CPR and ongoing pregnancy rate in the subgroup analysis for TSH threshold (Fig. 2). Only MR was significantly higher in the ATA positive and TSH > $2.5 \mu \mathrm{IU} / \mathrm{L}$ subgroup (Table 3).

In the univariate and multivariate logistic regression analysis, maternal age, TSH, AMH, number of retrieved oocytes, presence of ATA positivity, and the presence of at least one top-quality embryo were included to the regression model. In the last step, we found that only the presence of a top-quality embryo significantly affected the CPR with an OR of $1.732(p=0.03)$. Moreover, we performed an analysis to evaluate the effect of thyroid antibodies on the embryo quality. Although it did not reach any significance, the positivity of ATA had a negative impact on the development of at least one top-quality embryo compared to negative ATA groups (OR 1.447, $p=0.213$ ).

\section{Discussion}

In the present study, we evaluated the association between ATA and ART cycles. We did not observe any significant negative impact of ATA on pregnancy outcomes in euthyroid women undergoing GnRH antagonist ICSI cycles. However, fertilization rate was significantly lower and the presence of at least one top-quality embryo tended to be lower ratio in ATA-positive patients compared to ATAnegative patients. We also found that ATA positivity significantly increased the miscarriage rate in patients who have TSH levels above $2.5 \mathrm{IU} / \mathrm{L}$.

Thyroid dysfunction affects the reproductive health of women, and it becomes more apparent in patients undergoing assisted reproduction techniques [1]. Thyroid autoimmunity is an important cause for especially subclinical hypothyroidism, and its relationship with unexplained infertility, miscarriage, and maternal thyroiditis were studied in various studies $[6,10,11]$. Some studies showed that the presence of ATA was associated with the poor outcome in IVF cycles [12, 17]. Litwicka et al. found a strong association between poor IVF outcome and thyroid autoimmunity [18]. They also concluded that administration of prednisolone for affected thyroid autoimmunity may improve the pregnancy outcomes. On the contrary, many studies established no significant effect of thyroid autoimmunity on IVF cycle outcome $[10,19,20]$. In a recent study, Ke et al. have also demonstrated that thyroid autoimmunity had no impact on IVF/ICSI cycle outcome especially in women with diminished ovarian reserve [21]. Nevertheless, they found higher live birth rate in the women who had ATA positivity and normal ovarian reserve.

In the literature, there are controversial results regarding pregnancy outcome of patients who have positive ATA. Although Zhong et al. reported that women with positive ATA had significantly lower clinical pregnancy rate [17], other studies did not confirm this finding [19, $22,23]$. In two recent meta-analysis, authors observed similar clinical pregnancy rates between presence and absence of thyroid autoimmunity [24, 25]. We did not also observe any significant differences in terms of the clinical pregnancy rates among ATA-positive and ATA-

Table 1 Baseline characteristics of ATA-negative and ATA-positive patients

\begin{tabular}{|c|c|c|c|}
\hline Variable & ATA negative $(n=250)$ & ATA positive $(n=65)$ & $p$ \\
\hline Female age (years) & $31.4 \pm 4.5$ & $31.1 \pm 4.9$ & 0.47 \\
\hline $\mathrm{BMI}\left(\mathrm{kg} / \mathrm{m}^{2}\right)$ & $26.0 \pm 19.6$ & $25.8 \pm 5.7$ & 0.27 \\
\hline Duration of infertility (years) & $4.9 \pm 3.6$ & $5.0 \pm 4.3$ & 0.70 \\
\hline Basal FSH (mIU/mL) & $7.0 \pm 2.4$ & $6.9 \pm 3.2$ & 0.43 \\
\hline Basal $E_{2}(p g / m L)$ & $41.1 \pm 29.5$ & $49.3 \pm 22.9$ & 0.67 \\
\hline $\mathrm{AMH}(\mathrm{ng} / \mathrm{mL})$ & $4.3 \pm 3.0$ & $3.8 \pm 2.3$ & 0.34 \\
\hline TSH ( $\mu \mid \mathrm{IU} / \mathrm{L})$ & $1.9 \pm 0.8$ & $1.8 \pm 0.9$ & 0.44 \\
\hline Free $T_{3}(\mathrm{ng} / \mathrm{dL})$ & $3.2 \pm 0.4$ & $3.2 \pm 0.4$ & 0.86 \\
\hline Free $T_{4}(\mathrm{ng} / \mathrm{dL})$ & $1.3 \pm 0.2$ & $1.5 \pm 0.4$ & 0.001 \\
\hline
\end{tabular}

ATA anti-thyroid antibody, BMI body mass index, FSH follicle-stimulating hormone, $E_{2}$ estradiol, $A M H$ anti-mullerian hormone, $T S H$ thyroid-stimulationg hormone Values are mean \pm SD 
Table 2 Cycle outcomes of ATA-negative and ATA-positive patients

\begin{tabular}{llll}
\hline Variable & ATA negative $(n=250)$ & ATA positive $(n=65)$ & $p$ \\
\hline Total dose of gonadotropins (IU) & $1910.0 \pm 749.7$ & $2073.1 \pm 754.6$ & 0.09 \\
Peak estradiol $(\mathrm{pg} / \mathrm{mL})$ & $2069.1 \pm 1214.0$ & $2185 \pm 1273.1$ & 0.45 \\
Serum progesterone $(\mathrm{ng} / \mathrm{mL})$ & $0.75 \pm 0.52$ & $0.69 \pm 0.36$ & 0.74 \\
No. of retrieved oocytes & $8.3 \pm 3.9$ & $8.5 \pm 4.6$ & 0.87 \\
No. of metaphase 2 oocytes & $5.3 \pm 3.2$ & $5.6 \pm 3.5$ & 0.66 \\
Fertilization rate (\%) & $97.1 \pm 10.5$ & $91.5 \pm 19.8$ & 0.003 \\
Cleavage rate (\%) & $99.9 \pm 0.9$ & $97.7 \pm 13.8$ & 0.05 \\
Presence of top-quality embryo (\%) & 67.8 & 56.5 & 0.09 \\
Implantation rate (\%) & 50.4 & 50.8 & 0.95 \\
Miscarriage rate (\%) & 9.6 & 12.7 & 0.47 \\
\hline ATA anti-thyid
\end{tabular}

ATA anti-thyroid antibody

Values are mean \pm SD or (\%)

negative patients. However, a prospective case-control study revealed a significantly higher clinical pregnancy rate in antibody-positive women who had undergone IVF cycles ( $48 \%$ vs. $28 \%, p<0.05)$ [26]. Similarly, we observed a higher clinical pregnancy rate in ATA-positive women compared to negative cases, albeit not significantly so $(47.7 \%$ vs. $42.4 \%$, respectively, $p=0.44)$.

Van den Boogard et al. demonstrated that the presence of ATA increased the miscarriage rate in women with spontaneous pregnancy but they did not find this kind of raise in IVF pregnancies [6]. In a retrospective study, Zhong et al. found a higher miscarriage rate with ATA positivity $(26.9 \%$ vs. $11.9 \%$ compared to the control group, $p=0.002$ ) [17]. Also, Toulis et al. demonstrated a significant higher risk for miscarriage with ATA-positive compared with ATA-negative women undergoing IVF cycles [9]. Nevertheless, Sakar et al. did not find a significant impact of the presence of ATA on the miscarriage rate in a prospective case-control study [22]. In the present study, there was no significant difference regarding the miscarriage rate. Although there was a higher miscarriage rate in ATA-positive patients, this tendency did not reach any significance $(12.7 \%$ vs. $9.6 \%, p=0.47)$. In 2016, Busnelli et al. published a meta-analysis and they determined that the miscarriage rate was significantly higher in thyroid autoimmunity $(\mathrm{OR}=1.44, p=$ 0.02) [24]. They also found that TSH levels were higher in ATA-positive patients and assumed the association between the support role of thyroid dysfunction and pathophysiology of miscarriage. However, a recent metaanalysis by Poppe et al. failed to demonstrate this effect $(\mathrm{OR}=0.95, p=0.31)[25]$. The total events and studies

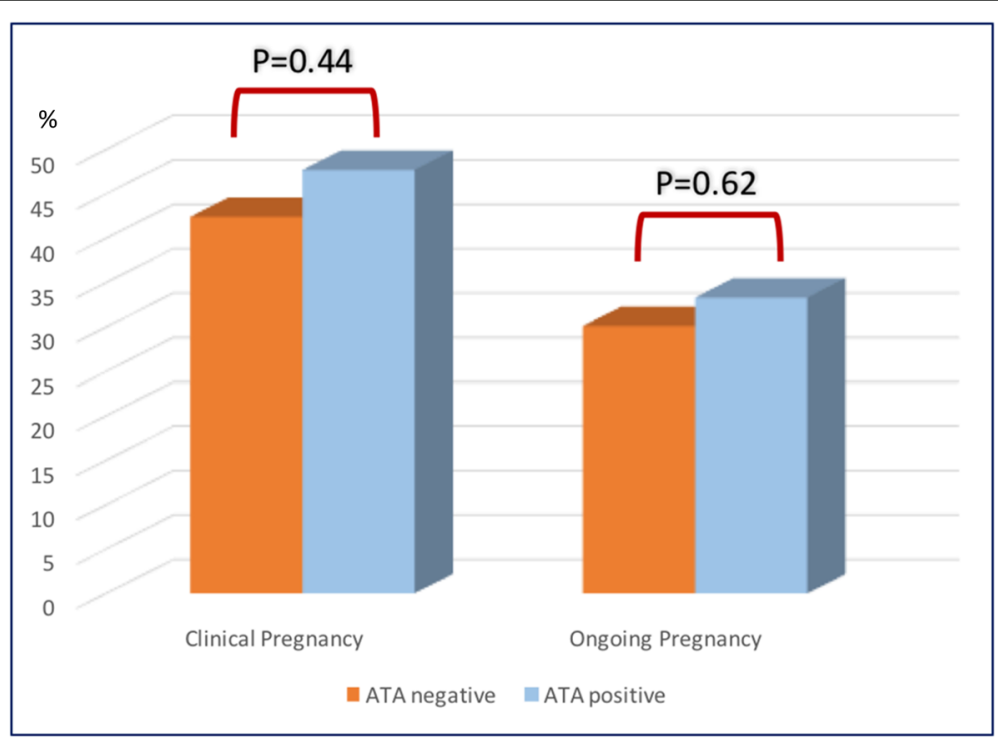

Fig. 1 The pregnancy outcomes of patients 
Table 3 Pregnancy outcome between negative and positive ATA for subgroup of TSH levels

\begin{tabular}{|c|c|c|c|c|c|c|}
\hline & \multicolumn{3}{|l|}{$\mathrm{TSH} \leq 2.5 \mathrm{IU} / \mathrm{L}$} & \multicolumn{3}{|l|}{$\mathrm{TSH}>2.5 \mathrm{IU} / \mathrm{L}$} \\
\hline & $\begin{array}{l}\text { ATA negative } \\
(n=174)\end{array}$ & $\begin{array}{l}\text { ATA positive } \\
(n=50)\end{array}$ & $p$ & $\begin{array}{l}\text { ATA negative } \\
(n=76)\end{array}$ & $\begin{array}{l}\text { ATA positive } \\
(n=15)\end{array}$ & $p$ \\
\hline Presence of top-quality embryo (\%) & 67.5 & 59.2 & 0.28 & 68.5 & 50 & 0.21 \\
\hline Implantation rate (\%) & 50.0 & 50.0 & 1 & 51.3 & 57.1 & 0.69 \\
\hline Miscarriage rate (\%) & 11.5 & 8.3 & 0.53 & 5.4 & 28.6 & 0.006 \\
\hline
\end{tabular}

ATA anti-thyroid antibody, TSH thyroid-stimulating hormone

included in Poppe's meta-analysis were lower compared to Busnelli's meta-analysis, so it might affect these results. Karacan et al. and Tan et al. also established that ATA positivity did not increase the risk of miscarriage in patients who had normal thyroid function $[19,27]$.

In a prospective study, ATA positivity did not have any impact on the ongoing pregnancy rate [22]. Karacan et al. did not observe any negative effect of ATA on ongoing pregnancy rate of euthyroid women who underwent ICSI cycles [19]. We also did not find any significant differences between ATA-positive and ATAnegative patients regarding the ongoing pregnancy rate. Some studies assessed the live birth rate, and most of them did not establish any significant difference in ATA-positive patients in comparison with ATA-negative patients [23, 25, 27]. Conversely, a meta-analysis reported a lower live birth rate with an OR $=0.65(p=$ 0.004) in thyroid autoimmunity [24]. Unuane et al. compared the cumulative live birth rate to predict the impact of thyroid autoimmunity, and they found similar cumulative live birth rate between thyroid autoimmunity and control groups [28]. They concluded that thyroid autoimmunity did not influence the cumulative live birth rates in patients undergoing IVF/ICSI cycles.
Two recent meta-analyses did not demonstrate a decrease in fertilization rate among IVF/ICSI cycles as well as a prospective study did not find any significant impact of thyroid autoimmunity on fertilization rate [19, 24, 25]. Nevertheless, Zhong et al. stated that the fertilization rate was significantly lower in ATA-positive women than those in ATA-negative women [17]. Similar to this study, we observed a lower fertilization rate in ATA-positive patients than the control group $(97.1 \pm 10.5$ vs. $91.5 \pm 19.8$, respectively, $p=0.003)$. Monteleone et al. investigated antithyroglobulin and anti-thyroperoxidase levels in both serum and follicular fluid [29]. They found that ATA levels in the follicular fluid were strongly correlated with serum levels and fertilization rate; moreover, top-quality embryos were significantly lower in ATA-positive women than in negative controls. They hypothesized that ATA may bind to the antigens expressed in the zona pellucida and may cause damage of zona pellucida, so it results to lower fertilization rate and decreased embryo quality. In the present study, we also assessed the rate of the topquality embryo between study groups. We did not find a significant difference but the rate of presence of topquality embryo was lower in ATA-positive patients compared with negative cases $(67.8 \%$ vs. $56.5 \%, p=0.09)$. Only

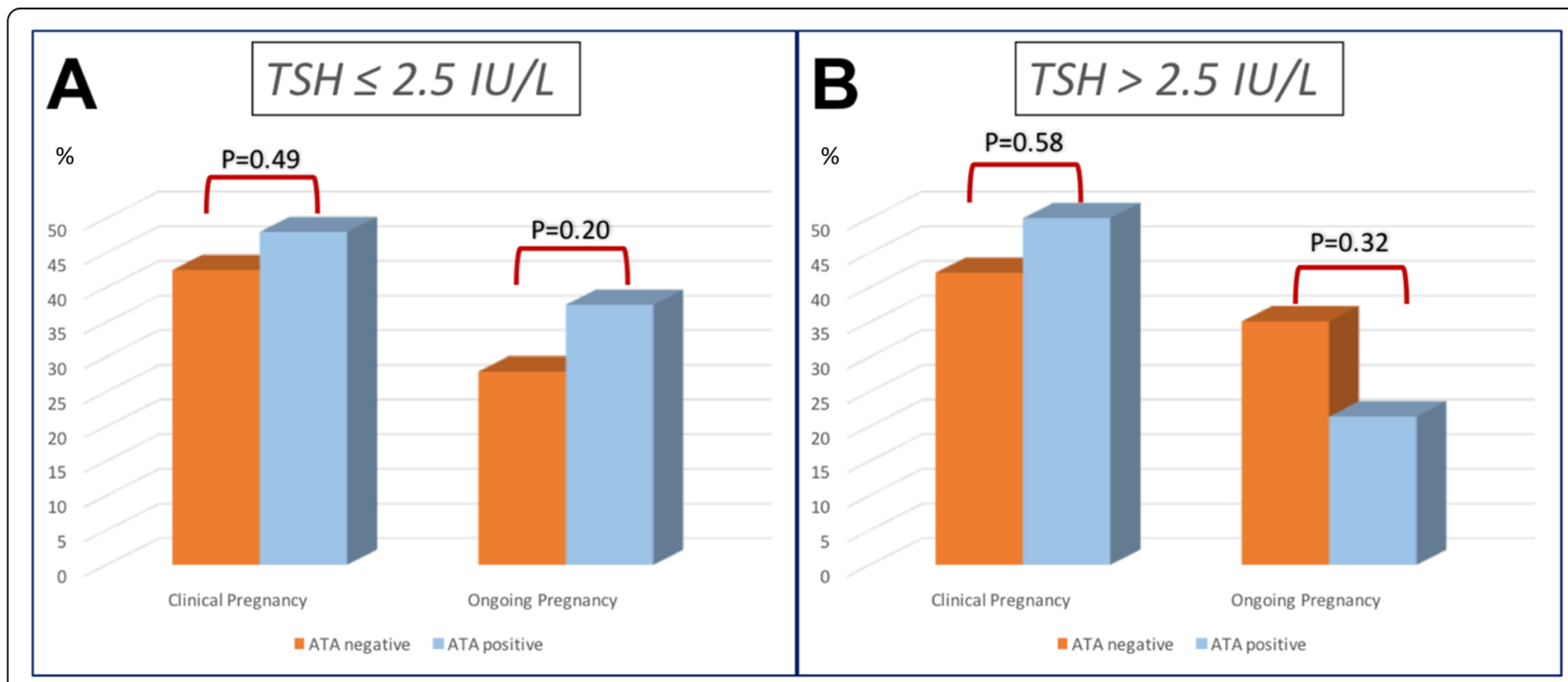

Fig. 2 The pregnancy outcomes of patients according to the TSH levels (a TSH $\leq 2.5 \mathrm{IU} / \mathrm{L}, \mathbf{b} \mathrm{TSH}>2.4 \mathrm{IU} / \mathrm{L}$ ) 
a few studies investigated the impact of ATA on embryo quality in assisted reproduction cycles. Kilic et al. found that embryo grade and the number of grade 1 embryos did not differ among ATA-positive and ATA-negative patients [12]. In a case-control study, it was suggested that anti-TPO status affects the embryo quality especially in euthyroid women with a TSH level $\leq 2.5 \mathrm{IU} / \mathrm{L}$ [13]. Andrisani et al. have performed a retrospective cohort study, and they concluded that thyroid autoimmunity affected embryo quality in euthyroid women undergoing IVF/ICSI cycles [14]. They also suggested that the results of the study support the hypothesis of Monteleone et al. From this point of view, ICSI was proposed to overcome this problem because ICSI did not require any interaction between the zona pellucida and the sperm cell. On the contrary, Weghofer et al. revealed a decreased embryo quality in women with thyroid autoimmunity even in those who underwent only ICSI cycles [13]. We performed ICSI procedure for all patients but we observed only a tendency on embryo quality in favor of ATA-negative cases $(p=0.09)$. Safarian et al. have also concluded that ICSI procedure is a preferred method in women with positive ATA [30].

We have performed a subgroup analysis to determine the effect of TSH threshold on the cycle outcomes. We defined this threshold as $2.5 \mathrm{IU} / \mathrm{L}$, according to the studies presented by Busnelli et al. and Weghofer et al. [13, 24]. In the subgroup analysis, the only miscarriage rate was significantly higher in ATA-positive women who had TSH $>2.5 \mathrm{IU} / \mathrm{L}$ than those of TSH $\leq 2.5 \mathrm{IU} / \mathrm{L}$. Similar to the total cohort, there were high rates of the topquality embryo in ATA-negative patients compared with positive in the subgroup evaluation. Unuane et al. have performed a threshold analysis with two TSH levels as 2.5 and $5 \mathrm{IU} / \mathrm{L}$ [28]. The cumulative delivery rates did not differ among ATA-positive and ATA-negative patients for the TSH level below 2.5 and 5 IU/L (45\% vs. $48 \%$ and $47 \%$ vs. $47 \%$, respectively). They also indicated that strict cut-off for TSH levels may cause some concerns such as overtreatment with levothyroxine in patients undergoing ART.

A recent guideline of American Society for Reproductive Medicine did not recommend universal thyroid screening before or during pregnancy and routine thyroid antibody testing is not recommended if there is no risk factors for thyroid dysfunction [31]. It is also recommended to treat patients especially if TSH $\geq 2.5 \mathrm{mIU} / \mathrm{L}$ with ATA or $>4$ $\mathrm{mIU} / \mathrm{L}$ in general. American Thyroid Association has published a guideline on thyroid disorders and pregnancy recently [32]. It is stated that there is no sufficient evidence whether a universal screening should be recommended for TSH concentrations before pregnancy, but they have made an exception for women planning assisted reproduction techniques and those determined positive ATA. However, a recent large-scaled retrospective cohort study showed that the worse pregnancy outcomes were determined in subclinical hypothyroidism compared to euthyroid pregnancies even in antibody negativity [33].

Strengths of our study were the uniform treatment modality used as GnRH antagonist protocol, only fresh embryo transfer cycles included. Some studies based on only anti-TPO analysis $[1,6,9,23]$. The importance of anti-TG was noticed in a recent guideline for thyroid disorders during pregnancy [32]. So, we evaluated both two types of ATA (anti-thyroglobulin and anti-thyroid peroxidase) and it is a strength of our study. Another crucial strength was consisting of the study population of only ICSI cycles.

The present study has some limitations. The important limitation was the retrospective nature of the study and a relatively small sample size. We have included more than one indication for ART cycles. Tan et al. restricted their population with male factor infertility, and they stated that it might avoid the selection bias [27]. However, we know that ICSI procedure is particularly successful in male factor infertility, so it may be considered another selection bias for this situation.

\section{Conclusion}

The presence of ATA did not have an impact on ICSI cycle outcome except the fertilization rate. Although it was not significantly different, embryo quality may be impaired with ATA positivity. The pregnancy outcomes of assisted reproduction techniques were not affected by thyroid autoimmunity but miscarriage rate slightly increases with thyroid autoimmunity. Fertilization rate and embryo quality are important determinants for ART cycles, and it was not clearly understood which mechanism of thyroid autoimmunity influences these parameters. Our study contributed to this conflicting field of the literature. Large-scale prospective randomized trials are required to reach more precise consequences.

\section{Abbreviations \\ ART: Assisted reproduction Technologies; IVF: In-vitro fertilization; TSH: Thyroid-stimulating hormone; ATA: Anti-thyroid autoantibodies; Anti- TG: Anti-thyroglobulin antibody; Anti-TPO: Anti-thyroid peroxidase antibody; ICSI: Intracytoplasmic sperm injection; FSH: Follicle-stimulating hormone; LH: Luteinizing hormone; AMH: Anti-mullerian hormone; OR: Odds ratio; Cl: Confidence interval; IR: Implantation rate; CPR: Clinical pregnancy rate; MR: Miscarriage rate}

\section{Acknowledgments \\ None}

\section{Authors' contributions}

VYT contributed to the study design, the data collection, the statistical analysis, the writing of the review, and the final revision. BI contributed to the data collection. ABT contributed to the writing of the review and final revision. All authors have read and approved the final manuscript.

Funding

None 


\section{Availability of data and materials}

The datasets used and/or analyzed during the current study are available from the corresponding author on reasonable request.

\section{Ethics approval and consent to participate}

The informed consent was not obtained from study participants because the present study was a retrospective data analysis. The study was approved by the Instutional Review Board and the principles of Helsinki Declaration were followed (Ref. Number: 2019/E.5275/342)

\section{Consent for publication}

All authors agreed for the publication at MEF journal.

\section{Competing interests}

The authors declare that they have no competing interest.

\section{Received: 16 October 2019 Accepted: 17 February 2020}

\section{Published online: 27 February 2020}

\section{References}

1. Krassas GE, Poppe K, Glinoer D (2010) Thyroid function and human reproductive health. Endocr Rev 31(5):702-755. https://doi.org/10.1210/er. 2009-0041

2. Krassas GE (2000) Thyroid disease and female reproduction. Fertil Steri 74(6):1063-1070. https://doi.org/10.1016/s0015-0282(00)01589-2

3. Poppe K, Glinoer D, Tournaye H, Devroey P, Velkeniers B (2008) Impact of the ovarian hyperstimulation syndrome on thyroid function. Thyroid 18(7): 801-802. https://doi.org/10.1089/thy.2007.0304

4. Artini PG, Uccelli A, Papini F, Simi G, Di Berardino OM, Ruggiero M, Cela V (2013) Infertility and pregnancy loss in euthyroid women with thyroid autoimmunity. Gynecol Endocrinol 29(1):36-41. https://doi.org/10.3109/ 09513590.2012 .705391

5. Poppe K, Glinoer D (2003) Thyroid autoimmunity and hypothyroidism before and during pregnancy. Hum Reprod Update 9(2):149-161. https:// doi.org/10.1093/humupd/dmg012

6. van den Boogaard E, Vissenberg R, Land JA, van Wely M, van der Post JA, Goddijn M, Bisschop PH (2011) Significance of (sub)clinical thyroid dysfunction and thyroid autoimmunity before conception and in early pregnancy: a systematic review. Hum Reprod Update 17(5):605-619. https:// doi.org/10.1093/humupd/dmr024

7. Poppe K, Velkeniers B, Glinoer D (2007) Thyroid disease and female reproduction. Clin Endocrinol 66(3):309-321. https://doi.org/10.1111/j.13652265.2007.02752.x

8. Prummel MF, Wiersinga WM (2004) Thyroid autoimmunity and miscarriage. Eur J Endocrinol 150(6):751-755

9. Toulis KA, Goulis DG, Venetis CA, Kolibianakis EM, Negro R, Tarlatzis BC, Papadimas I (2010) Risk of spontaneous miscarriage in euthyroid women with thyroid autoimmunity undergoing IVF: a meta-analysis. Eur J Endocrinol 162(4):643-652. https://doi.org/10.1530/EJE-09-0850

10. Negro R, Formoso G, Coppola L, Presicce G, Mangieri T, Pezzarossa A, Dazzi D (2007) Euthyroid women with autoimmune disease undergoing assisted reproduction technologies: the role of autoimmunity and thyroid function. J Endocrinol Investig 30(1):3-8. https://doi.org/10.1007/BF03347388

11. Bellver J, Soares SR, Alvarez C, Munoz E, Ramirez A, Rubio C, Serra V, Remohi J, Pellicer A (2008) The role of thrombophilia and thyroid autoimmunity in unexplained infertility, implantation failure and recurrent spontaneous abortion. Hum Reprod 23(2):278-284. https://doi.org/10.1093/humrep/ dem383

12. Kilic S, Tasdemir N, Yilmaz N, Yuksel B, Gul A, Batioglu S (2008) The effect of anti-thyroid antibodies on endometrial volume, embryo grade and IVF outcome. Gynecol Endocrinol 24(11):649-655. https://doi.org/10.1080/ 09513590802531112

13. Weghofer A, Himaya E, Kushnir VA, Barad DH, Gleicher N (2015) The impact of thyroid function and thyroid autoimmunity on embryo quality in women with low functional ovarian reserve: a case-control study. Reprod Biol Endocrinol 13:43. https://doi.org/10.1186/s12958-015-0041-0

14. Andrisani A, Sabbadin C, Marin L, Ragazzi E, Dessole F, Armanini D, Dona G, Bordin L, Ambrosini G (2018) The influence of thyroid autoimmunity on embryo quality in women undergoing assisted reproductive technology. Gynecol Endocrinol 34(9):752-755. https://doi.org/10.1080/09513590.2018. 1442427
15. Alpha Scientists in Reproductive M, Embryology ESIGo (2011) The Istanbul consensus workshop on embryo assessment: proceedings of an expert meeting. Hum Reprod 26(6):1270-1283. https://doi.org/10.1093/humrep/ der037

16. Gardner DK, Lane M, Stevens J, Schlenker T, Schoolcraft WB (2000) Blastocyst score affects implantation and pregnancy outcome: towards a single blastocyst transfer. Fertil Steril 73(6):1155-1158

17. Zhong YP, Ying Y, Wu HT, Zhou CQ, Xu YW, Wang Q, Li J, Shen XT, Li J (2012) Relationship between antithyroid antibody and pregnancy outcome following in vitro fertilization and embryo transfer. Int J Med Sci 9(2):121125. https://doi.org/10.7150/ijms.3467

18. Litwicka K, Arrivi C, Varricchio MT, Mencacci C, Greco E (2015) In women with thyroid autoimmunity, does low-dose prednisolone administration, compared with no adjuvant therapy, improve in vitro fertilization clinical results? J Obstet Gynaecol Res 41(5):722-728. https://doi.org/10.1111/jog. 12615

19. Karacan M, Alwaeely F, Cebi Z, Berberoglugil M, Batukan M, Ulug M, Arvas A, Camlibel T (2013) Effect of antithyroid antibodies on ICSI outcome in antiphospholipid antibody-negative euthyroid women. Reprod BioMed Online 27(4):376-380. https://doi.org/10.1016/j.rbmo.2013.07.002

20. Mintziori G, Goulis DG, Gialamas E, Dosopoulos K, Zouzoulas D, Gitas G, Venetis CA, Toulis KA, Kolibianakis EM, Tarlatzis BC (2014) Association of TSH concentrations and thyroid autoimmunity with IVF outcome in women with TSH concentrations within normal adult range. Gynecol Obstet Investig 77(2):84-88. https://doi.org/10.1159/000357193

21. Ke H, Hu J, Zhao L, Ding L, Jiao X, Qin Y (2020) Impact of thyroid autoimmunity on ovarian reserve, pregnancy outcome, and offspring health in euthyroid women following in vitro fertilization/intracytoplasmic sperm injection. Thyroid. https://doi.org/10.1089/thy.2018.0657

22. Sakar MN, Unal A, Atay AE, Zebitay AG, Verit FF, Demir S, Turfan M, Omer B (2016) Is there an effect of thyroid autoimmunity on the outcomes of assisted reproduction? J Obstet Gynaecol 36(2):213-217. https://doi.org/10. 3109/01443615.2015.1049253

23. Lukaszuk K, Kunicki M, Kulwikowska P, Liss J, Pastuszek E, Jaszczolt M, Meczekalski B, Skowronski K (2015) The impact of the presence of antithyroid antibodies on pregnancy outcome following intracytoplasmatic sperm injection-ICSI and embryo transfer in women with normal thyreotropine levels. J Endocrinol Investig 38(12):1335-1343. https://doi.org/ 10.1007/s40618-015-0377-5

24. Busnelli A, Paffoni A, Fedele L, Somigliana E (2016) The impact of thyroid autoimmunity on IVF/ICSI outcome: a systematic review and meta-analysis. Hum Reprod Update 22(6):775-790. https://doi.org/10.1093/humupd/ dmw019

25. Poppe K, Autin C, Veltri F, Kleynen P, Grabczan L, Rozenberg S, Ameye L (2018) Thyroid autoimmunity and intracytoplasmic sperm injection outcome: a systematic review and meta-analysis. J Clin Endocrinol Metab. https://doi.org/10.1210/jc.2017-02633

26. Muller AF, Verhoeff A, Mantel MJ, Berghout A (1999) Thyroid autoimmunity and abortion: a prospective study in women undergoing in vitro fertilization. Fertil Steril 71(1):30-34. https://doi.org/10.1016/s00150282(98)00394-x

27. Tan S, Dieterle S, Pechlavanis S, Janssen OE, Fuhrer D (2014) Thyroid autoantibodies per se do not impair intracytoplasmic sperm injection outcome in euthyroid healthy women. Eur J Endocrinol 170(4):495-500. https://doi.org/10.1530/EJE-13-0790

28. Unuane D, Velkeniers B, Deridder S, Bravenboer B, Tournaye H, De Brucker M (2016) Impact of thyroid autoimmunity on cumulative delivery rates in in vitro fertilization/intracytoplasmic sperm injection patients. Fertil Steril 106(1):144-150. https://doi.org/10.1016/j.fertnstert.2016.03.011

29. Monteleone P, Parrini D, Faviana P, Carletti E, Casarosa E, Uccelli A, Cela V, Genazzani AR, Artini PG (2011) Female infertility related to thyroid autoimmunity: the ovarian follicle hypothesis. Am J Reprod Immunol 66(2): 108-114. https://doi.org/10.1111/j.1600-0897.2010.00961.x

30. Safarian GK, Gzgzyan AM, Dzhemlikhanova Lyailya K, Niauri Dariko A (2019) Does subclinical hypothyroidism and/or thyroid autoimmunity influence the IVF/ICSI outcome? Review of the literature. Gynecol Endocrinol 35 (sup 1):5659. doi:https://doi.org/10.1080/09513590.2019.1653564

31. Practice Committee of the American Society for Reproductive M (2015) Subclinical hypothyroidism in the infertile female population: a guideline. Fertil Steril 104(3):545-553. https://doi.org/10.1016/j.fertnstert. 2015.05 .028 
32. Alexander EK, Pearce EN, Brent GA, Brown RS, Chen H, Dosiou C, Grobman WA, Laurberg P, Lazarus JH, Mandel SJ, Peeters RP, Sullivan S (2017) 2017 Guidelines of the American Thyroid Association for the Diagnosis and Management of Thyroid Disease During Pregnancy and the Postpartum. Thyroid 27(3):315-389. https://doi.org/10.1089/thy.2016.0457

33. Cakmak BD, Turker UA, Temur M, Ustunyurt E (2019) Pregnancy outcomes of antibody negative and untreated subclinical hypothyroidism. J Obstet Gynaecol Res 45(4):810-816. https://doi.org/10.1111/jog.13925

\section{Publisher's Note}

Springer Nature remains neutral with regard to jurisdictional claims in published maps and institutional affiliations.

\section{Submit your manuscript to a SpringerOpen ${ }^{\circ}$ journal and benefit from:}

- Convenient online submission

- Rigorous peer review

- Open access: articles freely available online

- High visibility within the field

- Retaining the copyright to your article

Submit your next manuscript at $\boldsymbol{\nabla}$ springeropen.com 